\title{
The effect of exercise and lifestyle interventions on heart rate variability in students at risk of cardiovascular disease - A pilot study
}

\author{
JO-ANNE H. KIRBY, C.C. GRANT, D.C. JANSE VAN RENSBURG AND R. COLLINS \\ Section Sports Medicine, University of Pretoria, P. Bag X 002, Hatfield, Pretoria, South Africa; \\ E-mail: jkirby@sun.ac.za
}

(Received: 14 November 2009 Revision Accepted: 8 March 2010)

\begin{abstract}
Cardiovascular disease is a major public health concern and it can be modified by diet, exercise and health awareness. However the impact of these interventions on the autonomic control of heart rate as measured by heart rate variability (HRV) is controversial. The aim of this study was to determine if indicators of HRV can be used to identify moderate risk of cardiovascular disease and to compare the influence of different lifestyle interventions in a student population. This was a double blind, randomised, prospective, pre-test, post-test group comparison. Thirty-seven university students at moderate risk of cardiovascular disease and 37 age matched controls, participated. HRV indicators as determined by time and frequency domain analysis were measured before and after interventions of exercise, diet, health awareness and a combination intervention. There were no significant pre-intervention differences between the healthy and test groups. Supine HRV parameters changed minimally in the health awareness and dietary groups during post testing. In the combined group, supine parameters were unchanged, however the standing parameters: standard deviation, root mean square successive difference and normalized high frequency decreased and normalized low frequency increased $(\mathrm{p}<0.05)$. HRV indicators of the other groups were unchanged. Analysis of HRV is insensitive to distinguish between healthy students and those at moderate risk of cardiovascular disease. However, HRV measured during an orthostatic stressor, was able to measure a significant parasympathetic withdrawal upon standing in the combined group indicating improved autonomic response to postural change in the combined intervention of health awareness, dietary adaptation and exercise.
\end{abstract}

Key words: Heart rate variability, lifestyle, risk, intervention.

\section{Introduction}

Cardiovascular disease is a major public health concern and even young people are developing risk factors through unhealthy lifestyles (Thomas, Baker \& Davies, 2003). The study of heart rate variability (HRV) offers insight into the autonomic control of the heart (Stein \& Kleiger, 1999). Reduced HRV (lower vagal tone) is associated with diabetes, hypertension, obesity, high cholesterol, physical inactivity, smoking and advanced age (American College of Sports Medicine (ACSM), 2006; Melo, Santos \& Silva, 2005; Thayer \& Lane, 2007). Modifying these risk factors reduces cardiovascular risk, morbidity and mortality (Thayer \& Lane, 2007). Exercise, diet and health awareness (Lie, 2007; Reddy \& Katan, 2004) are powerful tools in achieving this but the impact these interventions have on HRV are controversial. The influence of exercise on HRV has been inconclusive, specific aspects of diet have been studied (e.g. reducing salt), but a holistic picture has not been formed. The effect of counseling on lifestyle behavior has been assessed, but not in the light of HRV.

The aim of this pilot study was to determine if indicators of HRV can be useful in the identification of moderate risk of cardiovascular disease in a small group of students and to compare the influence of different lifestyle interventions in these students. The objective was to determine the effect on heart rate variability indicators, as measured by time and frequency domain analysis, before and after 16 week interventions of exercise, diet, health awareness and a combination intervention.

\section{Methods}


The sample group consisted of students of mixed ethnic origin, at moderate risk of cardiovascular disease from the University of the Western Cape in 2008. Moderate risk was defined as having two or more of the following: family history of heart disease, cigarette smoking, obesity, hypertension, hypercholesterolaemia, impaired fasting glucose and a sedentary lifestyle (ACSM, 2006); but no overt signs or symptoms of cardiovascular disease. Students were recruited during registration, a questionnaire was used to identify those potentially at risk and this was confirmed with biometric tests, blood glucose and cholesterol, exercise testing and blood pressure measurement. Excluded from the study were those with injury, illness or other ailment, with cardiovascular, pulmonary, metabolic, and/or orthopedic disease requiring medical attention or who use medication that could influence cardiovascular control. Ethical approval was obtained from the Ethical committee of University of Pretoria and informed consent was obtained from participants at the start of the study.

The first part of the study compared the baseline HRV of healthy (no risk of cardiovascular disease) students who were age, gender and race matched to the test group. For the second part, a double blinded randomized prospective pre-test, post-test group comparison - (randomised controlled trial) was performed. The "at risk" participants were enrolled in five randomly assigned groups of:

- control (maintained their normal lifestyle)

- health awareness (received motivational interviews and regular structured focus group discussions to educate and motivate various health concepts and lifestyle management skills without enforcing the application thereof (i.e. give them information and see what they do with it)

- dietary (a dietician constructed a healthy diet plan after a baseline eating log was kept)

- exercise (a structured exercise program at the biokinetics clinic) and a

- combination group which attended the health awareness, dietary and exercise groups.

After randomization within the test group, there were five in the control group, five in the exercise group, seven in the dietary group, eight in the health awareness and nine in the combined group. These groups were maintained for 16 weeks and then a follow-up of HRV measurement was taken. The assistants running the intervention groups and the participants were blinded to the existence of the other groups.

The HRV measurement was performed at the campus health clinic, in a quiet room with natural light during weekday mornings. Participants were requested not to perform any strenuous activity or overindulge in ethanol on the day before or on the test day. They were also requested to refrain from smoking and using caffeine or medication on the morning of the test and to eat a light breakfast before the test. The participants' height, weight, age and gender were recorded and body mass index calculated. The Polar RS800 strap and transmitter were applied using electrode gel to enhance sensitivity, while the tester held the watch within range. Recordings were taken at an accuracy of $1 \mathrm{~ms}$. Participants lay supine for 20 minutes breathing spontaneously without talking and subsequently stood up, leaning against the wall as an orthostatic stress test for 10 minutes. In order to reduce bias, the same watch was used for all the readings and the same assistant who was blinded to the groups, took the recordings.

The data was transferred to a computer, via infrared, directly on to the Polar Protrainer 5 software. On the tachogram, the last five supine minutes and the last five standing minutes were manually selected for interpretation. This allowed the heart rate to stabilize giving stationary data. In the literature, short segments of data have been found to provide comparable results to 24 h data (Bigger, Fleiss, Rolnitzky \& Steinman, 1993; Stein et al., 1999) and are characteristic to individuals as they are stable over months (Sinnreich, Kark, Friedlander, Sapoznikov \& Luria, 1998).

The filter power was set at moderate, with a minimum protection zone set at six beats per minute. The filter was only applied if there were noticeable abnormalities suggestive of poor contact (occasionally a problem 
in very thin girls where the chest strap was too big even on its smallest fitting). If there was an obvious section of poor contact it was manually selected and eliminated so that a continuous reading was available. The software interpreted the tachogram, producing time and frequency domain outputs which were then analyzed statistically.

In time domain analysis of HRV, the interval used for calculations is the time between consecutive $\mathrm{R}$ waves on the QRS complexes of the electrocardiogram; known as the R-R or the N-N interval. The smaller the standard deviation of the R-R interval, the lower the HR. Abbreviations are commonly used to denote these calculations: SDNN - standard deviation of all N-N intervals, RMSSD - the square root of the mean of the sum of the squares of differences between adjacent N-N intervals and pNN50 - the number of interval differences of successive $\mathrm{N}-\mathrm{N}$ intervals greater than $50 \mathrm{~ms}$ divided by the total number of $\mathrm{N}-\mathrm{N}$ intervals. The pNN50 and RMSSD represent parasympathetic modulation of the sino-atrial node (Grant \& Ker, 2008; Stein et al., 1999). Frequency domain analysis quantifies the variance (power) in the rhythm of the heart rates periodic oscillations, by grouping them into frequency bands viz. HF (high frequency) and LF (low frequency). Parasympathetic modulation is represented by HF whereas LF signifies both parasympathetic and sympathetic nervous control. Normalized LF power (LF nu) is calculated LF/LF+HF and reflects primarily sympathetic modulation of the heart rate, normalized HF power (HF nu) equals $\mathrm{HF} / \mathrm{LF}+\mathrm{HF}$ and represents vagal control. The LF/HF ratio reflects sympathovagal balance; an increase in the ratio suggests an increase in sympathetic modulation and a decrease, parasympathetic or both (Grant \& Ker, 2008; Stein et al., 1999).

\section{Statistical analysis}

For the comparison between the healthy and test groups in order to determine whether HRV can identify those at risk of moderate cardiovascular disease from those who are not, the Mann-Whitney test was used. A significant difference between the groups in time and frequency domain analysis was sought.

In the at risk test group, the pre-intervention, supine and standing results were compared and the distribution of each variable was assessed for distribution variance. The Kruskal-Wallis test was used to determine whether significant differences were present between the five groups at the beginning of the experiment and again at the post intervention test.

Wilcoxon Signed Rank tests were used to determine if significant changes took place within each group over time. In analysis of the results comparing post intervention to baseline, a significant change towards parasympathetic dominance reflected as an increase in SDNN, RMSSD, pNN50 and HF norm and a decrease in the LF/HF ratio and LF norm was sought. A p-value of $<0.05$ was considered significant.

\section{Results}

Apart from the expected body mass index (BMI) difference, there were no demographic differences between the healthy and at risk test groups before HRV testing. The demographic data of the two groups are represented in Table 1. After testing there were no significant differences in the HRV parameters between the at-risk group and the healthy control group.

There were no significant inter-group variations on the pre-test values (Kruskal-Wallis test) including gender, race, age and BMI of the five intervention groups. Therefore any intra-group changes are significant and not due to demographic differences between the groups. Table 2 summarizes the risk profile of the participants. Obesity is defined as a BMI of more than thirty $\mathrm{kg} / \mathrm{m}^{2}$.

Table 1: Group demographics 


\begin{tabular}{llllll}
\hline & \multicolumn{2}{c}{ Healthy control group } & & \multicolumn{2}{c}{ Test group } \\
\cline { 2 - 3 } \cline { 5 - 6 } & Male $(\mathrm{n}=5)$ & Female $(\mathrm{n}=32)$ & & Male $(\mathrm{n}=5)$ & $\begin{array}{l}\text { Female } \\
(\mathrm{n}=32)\end{array}$ \\
\hline Age (years) & $20.6 \pm 4.2$ & $23.7 \pm 8.1$ & & $20.6 \pm 4.2$ & $23.7 \pm 8.1$ \\
Height $(\mathrm{m})$ & $1.74 \pm 0.10$ & $1.61 \pm 0.07$ & & $1.74 \pm 0.09$ & $1.62 \pm 0.06$ \\
Weight $(\mathrm{kg})$ & $59.6 \pm 6.9$ & $59.4 \pm 11.7$ & & $87.8 \pm 26.4$ & $71.5 \pm 18.2$ \\
BMI $\left(\mathrm{kg} / \mathrm{m}^{2}\right)$ & $19.4 \pm 1.9$ & $22.5 \pm 3.1$ & & $28.66 \pm 6.2$ & $27.1 \pm 6.25$ \\
\hline BMI= body mass index & & & &
\end{tabular}

Table 2: Cardiovascular risk profile on entry to the trial

\begin{tabular}{lll}
\hline Risk factor & Number of participants & $(\%)$ \\
\hline Sedentary lifestyle & 33 & 89 \\
Smoking & 15 & 40 \\
Obesity & 12 & 32 \\
Family history of cardiovascular disease & 9 & 24 \\
Dyslipidemia & 6 & 16 \\
Risk factor & Number of participants & $(\%)$ \\
\hline
\end{tabular}

Intra group HRV evaluations with the Wilcoxon Signed Ranks test, revealed no change from pre-test to post-test in either the supine or standing parameters of the control group (Tables 3a \& 3b). However, within the supine pre-intervention to post- intervention of the other groups, the following small changes were found: in the dietary group, the LF nu increased from 0.391 to 0.615 ( $\mathrm{p}=0.043$ ), the HF nu decreased from 0.608 to $0.385(\mathrm{p}=0.043)$ and the $\mathrm{LF} / \mathrm{HF}$ ratio increased from 0.86 to $2.68(\mathrm{p}=0.043)$. The health awareness group's mean RR interval increased from $750 \mathrm{~ms}$ to $813 \mathrm{~ms}(\mathrm{p}=0.012)$ while the mean heart rate dropped from $80 \mathrm{bpm}$ to $74 \mathrm{bpm}$.

The combined group's supine parameters were unchanged (Table 3a \& 3b). The orthostatic test (standing) revealed no changes in the exercise, dietary or health awareness groups. However, many significant changes occurred in the combined group as represented in Table 4.

The standing values of mean RR and pNN50 decreased at a significance level of $10 \%$ and SDNN, RMSSD, HF, and HF norm decreased at a significance of $5 \%$. The LF/HF ratio and mean heart rate increased at a significance of $10 \%$ and the normalised LF increased at $5 \%$ significance.

\section{Discussion}

This study aimed to determine if HRV analysis was sensitive enough to identify moderate CVD risk. Results from this study indicated that HRV quantification was not able to indicate any differences between healthy students and those with moderate risk factors for cardiovascular disease.

The secondary aim of the study was to identify which lifestyle intervention if any, altered the HRV of the participants who were at moderate risk of CVD. The combined group of diet, health awareness and exercise showed significant changes. 
Table 3a: Supine HRV indicator mean values

Table 3b: HRV indicator mean values

\begin{tabular}{|c|c|c|c|c|c|c|c|c|c|c|c|c|c|c|c|}
\hline \multirow[b]{2}{*}{ HRV indicator } & \multicolumn{3}{|c|}{ Control Group } & \multicolumn{3}{|c|}{ Dietary Group } & \multicolumn{3}{|c|}{ Exercise Group } & \multicolumn{3}{|c|}{ Health Awareness Group } & \multicolumn{3}{|c|}{ Combined Group } \\
\hline & Pre & Post & $\mathrm{P}$ & Pre & Post & $\mathrm{P}$ & Pre & Post & $\mathrm{P}$ & Pre & Post & $\mathrm{P}$ & Pre & Post & $\mathrm{P}$ \\
\hline HR (bpm) & 90 & 92 & 0.498 & 85 & 89 & 0.400 & 90 & 93 & 0.078 & 97 & 95.0 & 0.483 & 89 & 97 & 0.075 \\
\hline $\mathrm{RR}(\mathrm{ms})$ & 683 & 660 & 0.500 & 704 & 684 & 0.499 & 676 & 659 & 0.343 & 617 & 632 & 0.401 & 681 & 623 & 0.066 \\
\hline SDNN & 58.3 & 60.7 & & 36.6 & & & 80.9 & 44.9 & 0.345 & 46.5 & 43.6 & & 49.9 & 31.7 & 0.028 \\
\hline RMSSD (ms) & 32.3 & 28.4 & 0.500 & 19.5 & 16.8 & 0.310 & 24.3 & 21.28 & 0.225 & 20.7 & 19.1 & 0.401 & 25.9 & 13.3 & 0.021 \\
\hline pNN50 (\%) & 6.66 & 4.06 & 0.225 & 1.46 & 1.3 & 0.600 & 3.16 & 2.66 & 0.273 & 2.11 & 1.56 & 0.128 & 3.644 & 1.13 & 0.091 \\
\hline LF nu & 0.753 & 0.762 & 0.893 & 0.664 & 0.798 & 0.176 & 0.736 & 0.758 & 0.686 & 0.771 & 0.731 & 0.575 & 0.716 & 0.833 & 0.028 \\
\hline HF nu & 0.247 & 0.238 & 0.893 & 0.335 & 0.202 & 0.176 & 0.263 & 0.242 & 0.686 & 0.229 & 0.269 & 0.575 & 0.283 & 0.167 & 0.028 \\
\hline LF/HF(\%) & 358 & 429 & 0.500 & 274 & 510 & 0.176 & 393 & 390 & 0.686 & 383 & 366 & 0.779 & 412 & 617 & 0.086 \\
\hline
\end{tabular}


Table 4: Combined interventions standing indicator

\begin{tabular}{lllcc}
\hline Variable & Pre test & Post test & p-value & Direction \\
\hline HR (bpm) & $89 \pm 9.6$ & $97 \pm 10.3$ & 0.075 & $\uparrow$ \\
RR (ms) & $681 \pm 76$ & $623 \pm 66$ & 0.066 & $\downarrow$ \\
SDNN (ms) & $50.0 \pm 19.5$ & $31.7 \pm 12.1$ & $* 0.028$ & $\downarrow$ \\
RMSSD (ms) & $25.9 \pm 12.5$ & $13.3 \pm 9.5$ & $* 0.021$ & $\downarrow$ \\
pNN50 (\%) & $3.6 \pm 4.2$ & $1.1 \pm 2.2$ & 0.091 & $\downarrow$ \\
LF nu & $0.72 \pm 0.16$ & $0.83 \pm 0.08$ & $* 0.028$ & $\downarrow$ \\
HF nu & $0.28 \pm 0.16$ & $0.17 \pm 0.08$ & $* 0.028$ & $\uparrow$ \\
LF/HF (\%) & $3.81 \pm 3.71$ & $6.30 \pm 2.78$ & 0.086 & $\downarrow$ \\
\hline$\uparrow$
\end{tabular}

$\uparrow=$ increased ; $\downarrow$ =decreased; *Statistical significant at $\mathrm{p}<0.05$.

The inability of HRV parameters to identify those at moderate risk of CVD may be influenced by the young age of the group examined. For example most smoking related illnesses only present in the 40s, these young people have not been exposed to their smoking or other risk factors for that long.

In the supine tests of the risk groups, there was a slight tendency towards increased sympathetic influence in the exercise and dietary groups and a decrease in the health awareness groups. In the orthostatic test, which is a physiological stimulus to enhance sympathetic drive to the sinoatrial node and withdraw parasympathetic, the response was significantly augmented in the combined group post intervention recordings but unchanged in the other groups.

It was expected that the dietary intervention should improve the HRV as hypertension, cholesterol, diabetes and obesity are all risk factors for CVD that can be addressed by diet (Reddy et al., 2004). Another study by Reddy et al. (2004) also found the nutritional habits of first-year students to be poor. However this study showed deterioration in three of the HRV parameters towards increased sympathetic dominance. Some of the participants in the health awareness group reported less smoking, smoking cessation, healthier eating as well as increased exercising. However these changes to healthier choices largely did not reflect in the HRV with only a reduction in the average heart rate and increase in the average R-R interval. All other parameters remained unchanged. Risky behaviours in youth are linked to unhealthy habits in adulthood (Werch et al., 2007). Most of the major CVD risk factors are lifestyle related and lifestyle counselling has been shown to be effective in causing change towards positive choices (Lie, 2007; Werch et al., 2007). However, this study showed that as an intervention providing only health information did not significantly influence HRV parameters. 
Within the exercise group, the normalised LF and HF also did not change indicating no influence on sympathovagal control. Various studies have been conducted to determine the association between HRV and exercise.

It is well known that physical exercise promotes a healthy heart but how exactly is not yet known. Literature suggests that the cardio-protective effect of training may be mediated by the "antiarrhythmic effect of increased vagal control of the heart,” (Buchheit, Simon, Piquart, Ehrhart \& Brandenberger, 2004).

Research on exercise training has revealed mixed results, there are studies showing that exercise improves HRV parameters and in other studies, HRV is uninfluenced by exercise. Differing methodology and confounding factors in the studies make comparison difficult (Grant et al., 2008). The one common factor in studies showing improved HRV (increased vagal dominance) was exercise of moderate intensity (Buchheit et al., 2004; Iwasaki, Zhang, Zuckerman \& Levine, 2003; Tulppo et al., 2003). The exercise intervention of this study was of a moderate intensity, strictly adhering to American College of Sports Medicine guidelines (ACSM, 2006) but despite this no improvement was seen.

It was likely that the combined group would show the greatest improvement in HRV because of their exposure to all the modalities, for example not only being given a diet, but understanding the importance of it from the counselling perspective. Results showed the values of the supine HRV indicators remained unchanged. However, the orthostatic stress test showed significant parasympathetic withdrawal and increased sympathetic influence on heart rate. This is reflected in the standing values as a decreased mean RR and pNN50 at a significance level of $10 \%$ and a decreased SDNN, RMSSD, HF, and HF norm at a significance of $5 \%$; and an increased $\mathrm{LF} / \mathrm{HF}$ ratio and mean heart rate at a significance of $10 \%$ and increased normalised LF at 5\% significance (Table 4). At first appearance it seems the effect of the combined intervention is the opposite of what was expected, however, studies that used orthostatic stressors have found similarities. Laitinen, Niskanen, Geelen, Länsimies and Hartikainen (2004), found a similar response when comparing the effect of orthostasis on young versus older people. The younger fitter group showed accentuated parasympathetic withdrawal and attenuated sympathetic activity in response to a head up tilt. The lack of response in the older group was attributed to age-related cardiovascular autonomic dysfunction. Gilder and Ramsbottom (2008), in a study on the autonomic response to postural stress in women with low and high volumes of exercise, encountered a similar finding, with the higher volume group having a far more significant parasympathetic withdrawal in response to standing than the less active group.

In this present HRV study, the apparent paradoxical effect of supposed worsening of the parameters in the combined groups orthostatic stressor may 
actually reflect an improved responsiveness / plasticity of the autonomic nervous system towards postural change in the combined group.

This pilot study was limited by the small group sizes which may account for some of the results. Care should be taken when interpreting HRV during an orthostatic stressor to accommodate the homeostatic reflex of increased sympathetic and decreased parasympathetic influence on heart rate during standing.

\section{Conclusion}

In this study analysis of HRV was not sensitive enough to distinguish between healthy students and those at moderate risk of cardiovascular disease. However, the orthostatic stressor elicited a significant parasympathetic withdrawal in the combined group indicating an improved autonomic response to postural change in the combined intervention of health awareness, dietary adaptation and exercise.

\section{Acknowledgements}

The authors would like to thank Marlize Alexander - statistical analysis, Lloyd Leach - screening methodology, Campus Health and Biokinetics clinics and Neana Gaffley for their assistance.

\section{References}

American College of Sports Medicine (2006). ACSM's Guidelines for Exercise Testing and Prescription ( $7^{\text {th }}$ ed). Philadelphia: Lippincott, Williams \& Wilkins.

Bigger, J.T., Fleiss, J.L., Rolnitzky, L.M. \& Steinman, R.C. (1993). The ability of several shortterm measures of RR variability to predict mortality after myocardial infarction. Circulation, 88(3), 927-934.

Buchheit, M., Simon, C., Piquard, F., Ehrhart, J. \& Brandenberger, G. (2004). Effects of increased training load on vagal-related indexes of heart rate variability: A novel sleep approach. American Journal of Physiology, Heart Circulation Physiology, 287, H2813-2818.

Gilder, M. \& Ramsbottom, R. (2008). Change in heart rate variability following orthostasis relates to volume of exercise in healthy women. Autonomic Neuroscience, 143, 73-76.

Grant, C.C. \& Ker, J.A. (2008). Autonomic response to exercise as measured by cardiovascular variability. South African Journal of Sports Medicine, 20(4), 102-107.

Irazusta, A., Hoyos, I., Irazusta, J., Ruiz, F., Diaz, F. \& Gil, J. (2007). Increased cardiovascular risk associated with poor nutritional habits in first-year university students. Nutrition Research, 27, 387-394. 


\section{Kirby, Grant, Janse Van Rensburg and Collins}

Iwasaki, K., Zhang, R., Zuckerman, J.H. \& Levine, B.D. (2003). Dose-response relationship of the cardiovascular adaptation to endurance training in healthy adults: how much training for what benefit? Journal of Applied Physiology, 95, 1575-1583.

Laitinen, T., Niskanen, L., Geelen, G., Länsimies, E. \& Hartikainen, J. (2004). Age dependency of cardiovascular autonomic responses to head-up tilt in healthy subjects. Journal of Applied Physiology, 96, 2333-2340.

Lie, D. (2007). Lifestyle counseling program may help reduce risk of developing type 2 diabetes. Diabetes Care, 30, 2465-2470.

Melo, R.C., Santos, M.D.B. \& Silva, E. (2005). Effects of age and physical activity on the autonomic control of heart rate in healthy men. Brazilian Journal of Medical and Biological Research, 38(9), 1331-1338.

Reddy, K.S. \& Katan, M.B. (2004). Diet, nutrition and the prevention of hypertension and cardiovascular diseases. Public Health Nutrition, 7, 167-186.

Sinnreich, R., Kark, J.D., Friedlander, Y., Sapoznikov, D. \& Luria, M. H. (1998). Five minute recordings of heart rate variability for population studies: repeatability and age-sex characteristics. Heart, 80, 156-162.

Stein, P.K. \& Kleiger, R.E. (1999). Insights from the study of heart rate variability. Annual Review Medicine, 50(1), 249-261.

Thayer, J.F. \& Lane, R.D. (2007). The role of vagal function in the risk for cardiovascular disease and mortality. Biological Psychology, 74(2), 224-242.

Thomas, N.E., Baker, J.S. \& Davies, B. (2003). Established and recently identified coronary heart disease risk factors in young people: The influence of physical activity and physical fitness. Sports Medicine, 33(9), 633-650.

Tulppo, M.P., Hautala, A.J., Mäkikallio, T.H., Laukkanen, R.T., Nissilä, S., Hughson, R.L. \& Huikuri, H.V. (2003). Effects of aerobic training on heart rate dynamics in sedentary subjects. Journal of Applied Physiology, 95, 364-372.

Werch, C.E., Bian, H., Moore, M.J., Ames, S., Di Clemente, C.C. \& Weiler, R.M. (2007). Brief multiple behavior interventions in a college student health care clinic. Journal of Adolescent Health, 41(6), 577-585. 